\title{
ELM induced divertor heat loads on TCV
}

\author{
J. Marki ${ }^{\mathrm{a}, *}$, R.A. Pitts ${ }^{\mathrm{a}}$, J. Horacek ${ }^{\mathrm{b}}$, D. Tskhakaya ${ }^{\mathrm{c}}$, The TCV Team

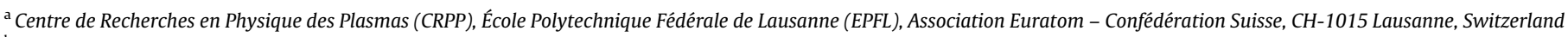 \\ ${ }^{\mathrm{b}}$ Institute of Plasma Physics, Association EUROATOM-IPP.CR, Za Slovankou 3, 18200 Prague 8, Czech Republic \\ ${ }^{\mathrm{c}}$ Association EURATOM-ÖAW, Institut für Theoretische Physik, A-6020 Innsbruck, Austria
}

\section{A R T I C L E I N F O}

\section{PACS:}

52.40.Hf

52.55.Fa

52.55.Rk

$52.70 . \mathrm{Kz}$

\begin{abstract}
A B S T R A C T
Results are presented for heat loads at the TCV outer divertor target during ELMing H-mode using a fast IR camera. Benefitting from a recent surface cleaning of the entire first wall graphite armour, a comparison of the transient thermal response of freshly cleaned and untreated tile surfaces (coated with thick codeposited layers) has been performed. The latter routinely exhibit temperature transients exceeding those of the clean ones by a factor $\sim 3$, even if co-deposition throughout the first days of operation following the cleaning process leads to the steady regrowth of thin layers. Filaments are occasionally observed during the ELM heat flux rise phase, showing a spatial structure consistent with energy release at discrete toroidal locations in the outer midplane vicinity and with individual filaments carrying $\sim 1 \%$ of the total ELM energy. The temporal waveform of the ELM heat load is found to be in good agreement with the collisionless free streaming particle model.
\end{abstract}

(c) 2009 Elsevier B.V. All rights reserved.

\section{Introduction}

Fast infra-red (IR) cameras are being increasingly employed on tokamaks as a tool to study the physics and consequences for first wall and divertor target surfaces (in terms of heat loads) of transient events such as edge localised modes (ELMs) and disruptions. Concerning ELMs, which will be the focus of this contribution, quantities of interest are, for example, the timescales of the ELM event (e.g. rise times to peak heat flux) on the divertor targets [1], the extent to which deposition profiles are broadened during the ELM compared with the inter-ELM periods and the presence of filaments in the profile (and if they are present, the energy fraction that they carry) [2]. In addition, an important diagnostic issue is the influence of surface layers, present in any real tokamak experiment, on bulk target material and the consequences of these layers for correct interpretation of the heat fluxes deduced from the IR surface temperature measurements [3,4]. A new fast IR diagnostic, viewing the outer divertor target, has been installed on the TCV tokamak and has recently provided first data for transient interactions in ELMing ohmic H-mode, of which Type-IIIs are shown in this article. Results are presented here for fast, sub-array measurements of power deposition profiles and ELM rise times on the $150-300 \mu$ s timescale, with a temporal shape in reasonable agreement with an analytic expression based on the assumption of particles free streaming to the divertor target from an upstream

\footnotetext{
* Corresponding author. Present address: EPFL SB CRPP, Bâtiment PPB, Station 13, CH-1015 Lausanne, Switzerland.

E-mail address: janos.marki@epfl.ch (J. Marki).
}

release point [1]. In addition, unambiguous observations of ELM filament heat deposition on the targets and unique observations of differences in transient surface temperature response on neighbouring surfaces with and without thick co-deposited layers will be presented.

\section{Experiment}

Fig. 1 illustrates the implementation of the fast, vertically viewing IR diagnostic on TCV imaging the outer divertor target area, together with a photograph of the vacuum vessel floor and a corresponding image as seen by the IR camera. The device itself is a Thermosensorik CMT256 M HS, with a $256 \times 256$ pixel CMT focal plane array (FPA) detector sensitive in the $1.5-5.1 \mu \mathrm{m}$ wavelength range [5]. The camera acquisition frequencies are in the range $880 \mathrm{~Hz}$ (full frame) $-25 \mathrm{kHz}$ (sub-array) with integration times from $1.076 \mathrm{~ms}$ down to $1 \mu \mathrm{s}$. In sub-array mode of operation, the resulting time resolution down to $\sim 40 \mu$ s is sufficient to supply several points during the ELM rise phase for TCV Type-III ELMs, enabling individual events to be studied and avoiding the coherent averaging required when using slower systems. The relay optics consists of $1 \mathrm{Ge}$ and $6 \mathrm{Si}$ anti-reflection coated lenses arranged in three groups imaging the vessel floor directly onto the FPA. This represents an upgrade from a preliminary, similar arrangement (from which a selection of results were reported in [6]) consisting entirely of Ge lenses with a smaller FOV and poorer image quality.

The standard data analysis procedure consists of converting the 14-bit digital IR signal into a relative photon intensity based on a pixel-by-pixel calibration. This data is then averaged in the toroidal 

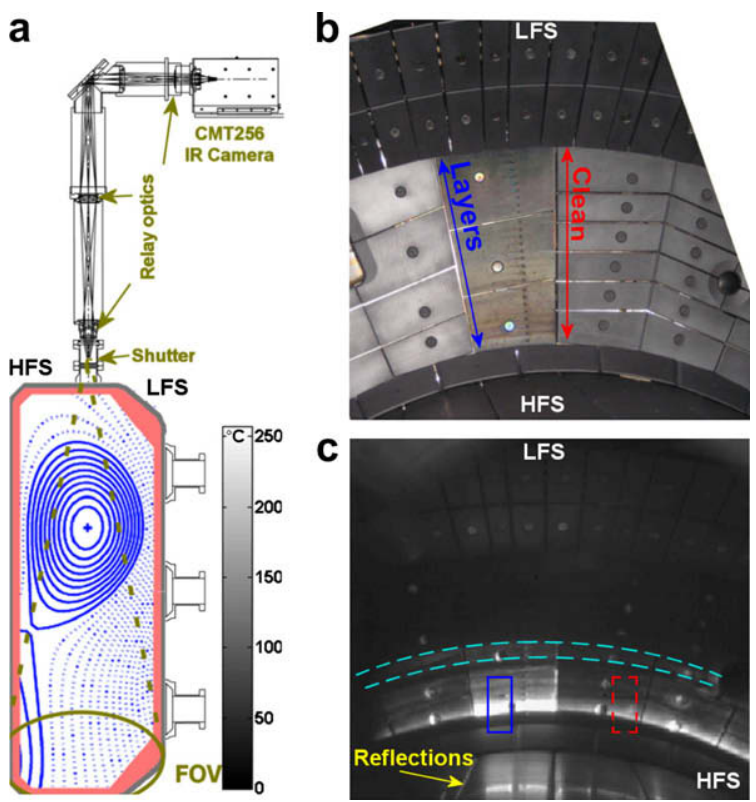

Fig. 1. (a) The vertically viewing fast IR diagnostic. (b) A photo taken just before the restart of the 2008 TCV campaign. The three large tiles in the centre (labelled 'Layers') are the untreated tiles containing the outer divertor Langmuir probe array. (c) IR camera image (\#35033). Note how the untreated tiles heat up to considerably higher temperatures. The coloured boxes represent the areas used in faster, subarrayed recordings. A filamentary deposition pattern can be observed (example highlighted by cyan dashed line). The central column reflects the IR radiation emanating from the strike point. (For interpretation of the references to colour in this figure legend, the reader is referred to the web version of this article.)

direction (over approximately $3.5^{\circ}$ ), resulting in radial profiles of photon intensity as a function of time. The latter are subsequently converted into radial profiles of surface temperature assuming an emissivity of 0.85 . Heat flux profiles are then determined using the 2D finite difference code THEODOR [7], including temperature dependent thermal properties of the polycrystalline graphite target tiles, together with a simple model for the thin layers present on the tile surfaces [4]. Whilst these thin deposited layers complicate the derivation of heat fluxes from surface temperatures, they are also quite useful since the temperature rise on a layered surface can exceed that of clean graphite surfaces by a factor of 3 for the same incident heat flux. This improves signal to noise and allows the detection of filamentary structures in the heat deposition.

During the recent (2007), year-long TCV shutdown (February 2007-March 2008), all 1600 polycrystalline graphite armour tiles have been removed and their surfaces grit-blasted with fine $(\sim 60 \mu \mathrm{m}) \mathrm{B}_{4} \mathrm{C}$ particles. Although the primary aim was to eliminate the frequent problems with dust (due to co-deposited layer spallation) experienced in discharges prior to the opening, this surface cleaning process offers an interesting possibility to study the effect of layers on the transient response observed with the IR system. To this end, the three tiles housing the outer divertor Langmuir probe array were left untreated (Fig. 1(b)). Although the thickness of the layers on these tiles has not been measured, they are the result of $\sim 8$ years of TCV operation, including $\sim 15$ vessel boronisations. The in-experiment deposition has never been measured on TCV, however, each boronisation accounts for $\sim 0.1 \mu \mathrm{m}$ of boron rich carbon layers, deposited uniformly on the first wall. The likely thickness is of the order of several microns.

The camera's nearly linear response with photon flux represents a challenge for ELM studies, since these fluxes can vary by 3 or more orders of magnitude for the typical temperature ranges of TCV divertor target surfaces during ELMs (from $25^{\circ} \mathrm{C}$ up to $700{ }^{\circ} \mathrm{C}$ ). This, together with a high sensitivity to electronic drifts at low signal values, prevents the inter-ELM and ELM phases from being fully resolved with a single integration time setting. As a consequence, a series of similar discharges have been used to focus on separate issues with appropriate choice of camera parameters. The pulses are ohmically heated, single null lower ELMing Hmodes in forward field (see magnetic equilibrium in Fig. 1(a)) with $I_{\mathrm{p}}=380 \mathrm{kA}, B_{\mathrm{T}}=1.43 \mathrm{~T}, \bar{n}=4.3 \cdot 10^{19} \mathrm{~m}^{-3}$, plasma stored energies of $W \sim 15 \mathrm{~kJ}$, ELM frequencies $f_{\mathrm{ELM}}=90-120 \mathrm{~Hz}$ and $\Delta W_{\mathrm{ELM}} /$ $W \sim 5-10 \%)$. Inter-ELM profiles have been measured in full frame mode with an integration time $\tau_{\text {int }}=101.1 \mu$ s whilst sub-arrays $(40 \times 16$ pixel $)$ with short integration times have been used to acquire data at higher frequency and thus resolve the individual ELMs. This sub-array has been placed at first on a 'clean' tile in the FOV $\left(\tau_{\text {int }}=15.3 \mu \mathrm{s}\right)$ and then on the untreated surface of the innermost Langmuir Probe tile $\left(\tau_{\text {int }}=4.8 \mu \mathrm{s}\right)$ in a subsequent discharge (see Fig. 1(c)).

\section{ELM filaments}

Evidence for filamentary heat deposition in the IR view is occasionally recorded during the ELM rise phase. We have observed this in $\sim 10 \%$ of ELMs recorded thus far, though there is as yet insufficient data to draw a general conclusion. To study the filament spatial distribution requires the full frame operation mode (FOV with toroidal extent $\sim 45^{\circ}$ ) and therefore limited time resolution (2-3 frames per ELM). In this case, only a few events are found in which the frame time coincides with the instant of filament heat deposition, but it is very likely that many more pass undetected inbetween the sampling points. An example is shown in Fig. 2(d). Several distinct filaments can be seen with almost purely toroidal inclination above the main band of emission at the strike point. Such patterns can be qualitatively reproduced by field line tracing calculations following the approach in $[2,8]$. Assuming: (1) a filament release point in the outboard midplane SOL, (2) that the pre-ELM magnetic field structure is not disturbed by the filament [9] and (3) that there is no significant poloidal rotation of the filament, then the equilibrium reconstruction can be used to trace field lines from the point of origin to the divertor targets. This has been performed to produce the plot in Fig. 2(a), where a single filament, assumed to extend $2.5 \mathrm{~cm}$ radially across the midplane SOL, has been traced to the outer divertor. In Fig. 2(b), this process is extended to six toroidally equidistant filaments with $2.5 \mathrm{~cm}$ radial extent. In Fig. 2(c), an appropriate portion of Fig. 2(b) has been extracted and placed above the IR image in Fig. 2(d), demonstrating qualitative agreement between the simple model and experiment and indicating an ELM with toroidal mode number $n=6$. A picture of multiple filaments released in the midplane region during the ELM evolution, as seen elsewhere (e.g. AUG [2], MAST [8]) is therefore also consistent with these early TCV observations.

In sub-array mode, spatial information on the filament patterns is lost, but the filament evolution at a single toroidal location can be followed. As these filaments are infrequently glimpsed in the fast acquisition mode, it follows that they must contain only a low fraction of the energy deposited by the main, toroidally symmetric heat flux in the strike point vicinity. When they are found, a very rough, preliminary estimate of the energy within single filaments (the calculation is performed by assuming a toroidally symmetric ring and isolating the filament-associated minor peaks in the heat flux from the main ELM profile) amounts to at most a few 10 's of Joules, of the order of $1 \%$ of $\Delta W_{\mathrm{ELM}}$. Note that this has been performed on a preliminary, limited dataset of $\sim 10$ filaments, so this value could change as we build a larger database and get more measurements of the toroidal variation of filament energy deposition. During the radial propagation, at some point the filaments are connected to the targets in the parallel direction, 

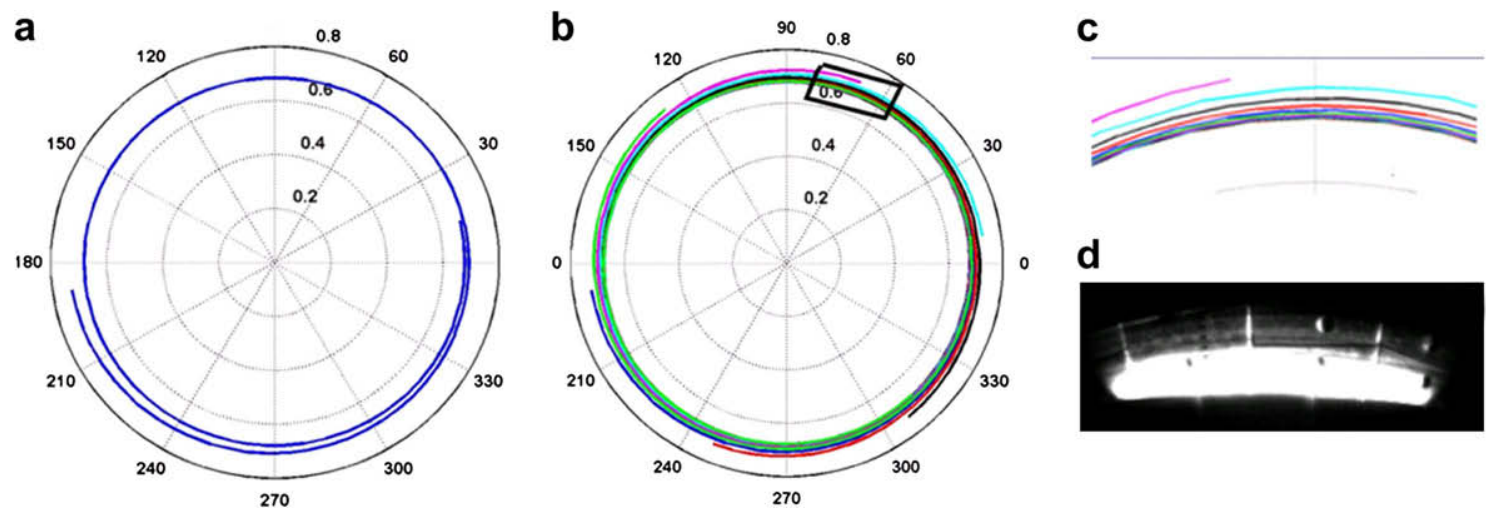

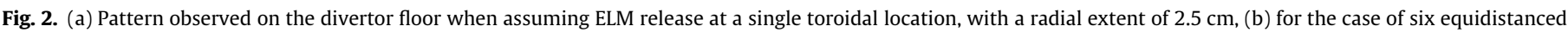

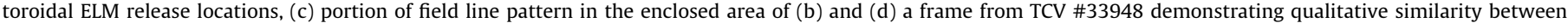
experiment and the field line tracing. (For interpretation to colours in this figure, the reader is referred to the web version of this paper.)

gradually loosing their energy as they continue their propagation, which means that as different filaments will be deposited at different toroidal locations, the energy content in a single filament as observed by a spatially restricted diagnostic such as the IR will vary considerably - however, once the dataset is sufficiently large, we can expect to have sufficient measurements at each phase of the filament-target interaction to be able to quantify the deposited heat load by individual filaments with an acceptable statistical uncertainty.

\section{Heat deposition characteristics}

In the presence of a surface layer on a bulk material, the increased surface temperature seen during a transient event compared with a clean surface (up to a factor 3 higher, see Fig. 3) can be accounted for in a simple way by assuming a layer with zero heat capacity and using a heat transmission coefficient $\alpha$ [4]. In this case, the bulk temperature is related to the measured IR surface temperature by $T_{\text {bulk }}=T_{\mathrm{IR}}-q / \alpha$, with $q$ the incident heat flux density. Thus, a smaller $\alpha$ implies a stronger layer effect. In estimating the power fluxes during the ELMs, $\alpha$ is varied within the THEODOR
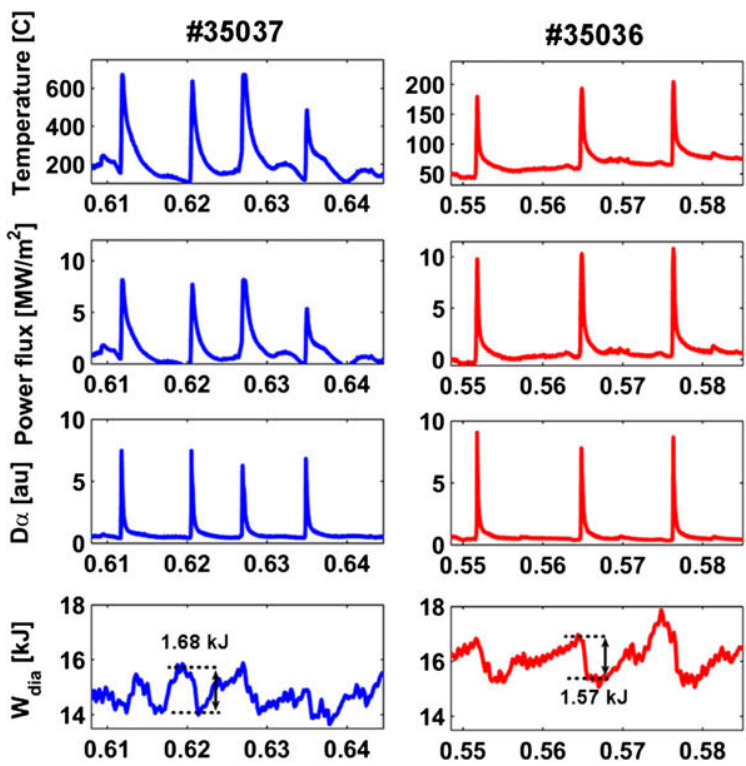

Fig. 3. Comparison of ELMs observed on a clean graphite (right) and layer-covered (left) tile. Despite similar ELM energies, the temperature rise to the left is a factor $\sim 3$ higher. calculations until the negative spikes are eliminated in the temporal and spatial transients (too low an $\alpha$ causes systematic underestimation of the peak heat flux, hence the highest alpha value not yielding negative heat fluxes is used). For ELMs on the cleaned and untreated tiles, $\alpha_{\text {clean }}=85000 \mathrm{~W} / \mathrm{m}^{2} \mathrm{~K}$ and $\alpha_{\text {layer }}=15000 \mathrm{~W} /$ $\mathrm{m}^{2} \mathrm{~K}$, respectively have been extracted. That $\alpha_{\text {clean }}$ is not essentially infinite is an indication that some degree of layer accumulation occurred already during the $\sim 10$ days of restart operation before the measurements described here were made. In fact, this is confirmed by visual inspection during a short vacuum opening several weeks after the beginning of restart. In the photograph shown in Fig. 4 (taken at approximately normal incidence), which includes the IR camera FOV, regions of co-deposition can clearlybe seen as a blue tinge on the cleaned tiles. Using the interference colour method developed by Wienhold and Littmark [10], a bluish hue could correspond approximately to carbon co-deposited layer thicknesses in the ranges $60-100 \mathrm{~nm}$ and $180-200 \mathrm{~nm}$ for the first two interference bands (if the carbon tile substrate can be assumed to behave similarly to the Si substrates for which the thicknesses derived in [10] apply). Such values are not inconsistent with the layer thick-

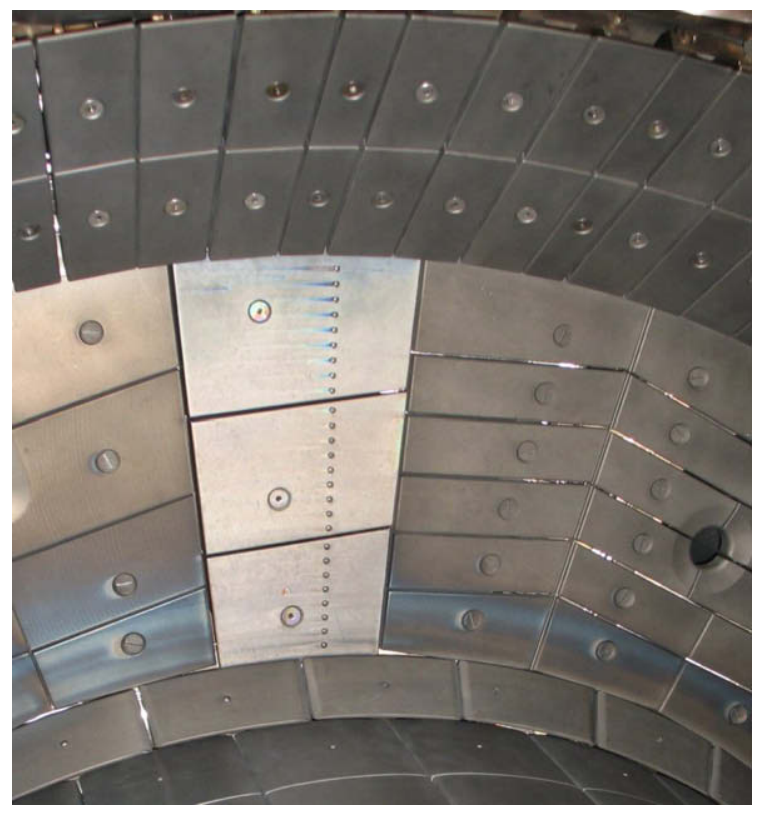

Fig. 4. Freshly developed layers around the strike point region (not visible on the untreated tiles due to the absence of interference patterns). (For interpretation to colours in this figure, the reader is referred to the web version of this paper.) 
Table 1

Averaged ELM characteristic values from TCV shots \#35033, \#35036 and \#35037.

\begin{tabular}{lcl}
\hline & $q_{\max }\left(\mathrm{MW} / \mathrm{m}^{2}\right)$ & FWHM $(\mathrm{cm})$ \\
\hline ELM (clean) & 10.8 & 2.1 \\
Inter-ELM (clean) & 0.8 & 0.9 \\
ELM (layers) & 8 & 1.8 \\
Inter-ELM (layers) & 1.2 & 0.7 \\
\hline
\end{tabular}

ness which could have accumulated within the few week restart phase (during which diverted plasmas with strike points on the vessel floor formed only part of the total number of pulses executed).

Table 1 compiles characteristic values for the maximum heat flux and the full width at half maximum (FWHM) for the ELM and inter-ELM profiles measured on the different tile surfaces. Unfortunately, there are thus far insufficient discharges for a detailed statistical analysis or a systematic layer/clean surface comparison. Nevertheless, this limited dataset suggests that for these Type-III ELMs, the ELM heat flux profile width is approximately doubled compared to that in the inter-ELM phases.

\section{Timescales for heat deposition}

The high acquisition frequency of the IR camera in sub-array mode allows the time dependence of the ELM heat flux to be studied in detail on an event-to-event basis, allowing comparison, for example, with the time evolution predicted on the basis of kinetic modelling. An example from TCV is shown in Fig. 5. Sophisticated 1D Particle-in-cell (PIC) simulations of the ELM pulse propagation along the magnetic field in the SOL are now becoming available [11] and are in fact underway for the TCV ELMs described here, but are insufficiently mature for inclusion in this contribution. Instead, a comparison with an analytic expression derived in [12] (and used also for experiment-model comparison of IR heat fluxes on JET and ASDEX Upgrade [13]) has been performed. This model is based on the assumption of a delta-function release of collisionless Maxwellian-distributed particles at the midplane, followed by free streaming of the expelled particles to the targets.According to this model [12], the time dependence of the target heat flux, $q_{\mathrm{ELM}}$, is given by:

$q_{\mathrm{ELM}}(t)=\frac{E_{\mathrm{ped}}}{\sqrt{\pi} \tau_{\mathrm{FSP}}} \cdot \exp \left[-\left(\frac{\tau_{\mathrm{FSP}}}{t}\right)^{2}\right] \times\left(\frac{\tau_{\mathrm{FSP}}}{t}\right)^{2} \times\left[1+\left(\frac{\tau_{\mathrm{FSP}}}{t}\right)^{2}\right]$

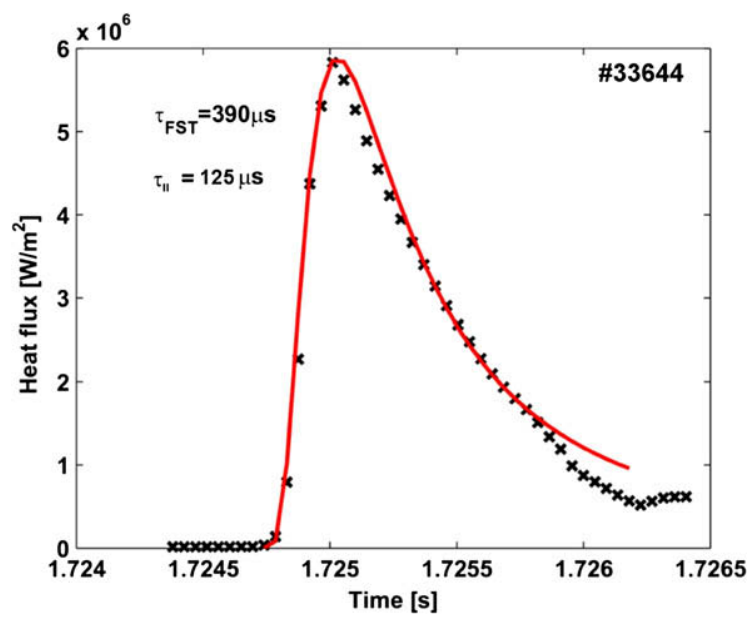

Fig. 5. Experiment to free streaming particle model comparison for the peak heat flux evolution of a $1.7 \mathrm{~kJ}$ ELM. where $E_{\text {ped }}$ is the pedestal stored energy and $\tau_{\mathrm{FSP}}$, the free streaming particle timescale. Even given the relatively high collisionality of the TCV pedestal in these ohmic H-modes $\left(v^{*} \sim 0.7\right)$, this expression, based on a collisionless approximation, provides a reasonable fit to the temporal evolution of TCV type-III ELMs, for the proper choice of $\tau_{\mathrm{FSP}}$. For the example presented an in Fig. $5, \tau_{\mathrm{fsp}}=390 \mu$ s is derived, a value that is high when compared solely to the parallel connection time, which is $\tau_{\|} \sim 125 \mu$ s for this discharge. This means that whilst there is qualitative agreement with the analytic model, the value is too large and cannot be interpreted solely as a parallel connection time. Possible candidates influencing this quantity could be the finite duration of the ELM event itself (of the order $\sim 100 \mu \mathrm{s}$ ) or the pedestal rotation. The effect of the finite ELM duration can be investigated by PIC simulations, and the simultaneous pedestal rotation measurement will be included in the future experimental programme on TCV.

\section{Conclusions}

A new fast IR imaging diagnostic, viewing the TCV outer divertor target has been successfully commissioned and exploited to make measurements of transient heat deposition during Type-III ELMs. Filamentary power deposition has been seen during the rise phase of ELM power deposition with full frame capture of an $\sim 45^{\circ}$ toroidal extent showing several filaments at radial locations in the SOL beyond the symmetric band of intense heat flux in the strike point vicinity. This distribution can be qualitatively matched by a simple model assuming filament expulsion at the outboard midplane at a number of toroidally discrete locations with radial extent $\sim 2 \mathrm{~cm}$. At the same time, in discharges in which the camera is operated at higher acquisition frequencies (at the cost of reducing the image to a sub-array), a small set of observations of the filamentary heat deposition temporal evolution at a small fraction of the toroidal circumference suggest that the energy content of individual filaments is only $\sim 1 \%$ of the total plasma stored energy drop due to the ELM, however, this value needs to be refined with the recording of a larger dataset in the future.

The measurements reported here followed a long tokamak shutdown, during which the front surface of all first wall graphite armour tiles, with the exception of three in the IR camera FOV, were grit-blasted to remove co-deposited layers. A pronounced difference in the ELM transient temperature response is observed between the cleaned and untreated tiles, demonstrating the important influence of thermally decoupled thick layers for fast IR thermography. An analytic expression derived in [12] on the basis of Maxwellian particles released upstream by the ELM and propagating collisionlessly to the targets provides a qualitative, but not quantitative agreement with the measured time evolution of the divertor heat flux.

\section{Acknowledgement}

This work was supported in part by the Swiss National Science Foundation and EURATOM.

\section{References}

[1] T. Eich et al., JNM 337-339 (2005) 669.

[2] T. Eich et al., PRL 91 (2003) 195003.

[3] P. Andrew et al., JNM 337-339 (2005) 99.

[4] T. Eich et al., PPCF 49 (5) (2007) 573-604.

[5] For further information on the detector, please visit the manufacturer's website: www.thermosensorik.com.

[6] J. Marki et al., EPS2007 Europhysics Conference Abstracts, vol. 31F P1.053 2007.

[7] A. Herrmann et al., JNM 313-316 (2003) 759-767.

[8] A. Kirk et al., PPCF 49 (8) (2007) 1259-1275. 
[9] M. Jakubowski, J. Nucl. Mater. 390-391 (2009) 781.

[10] P. Wienhold, U. Littmark, E-MRS Symposium Proceedings, vol. XVII,

[11] D. Tskakhaya, these Proceedings.

Strasbourg, France, 1987, p. 441

[12] W. Fundamenski et al., PPCF 48 (1) (2006) 109-156.

[13] T. Eich et al., J. Nucl. Mater. 390-391 (2009) 760. 